ACCEPTED MANUSCRIPT

\title{
Bifurcations in synergistic epidemics on random regular graphs
}

To cite this article before publication: Sergei N Taraskin et al 2019 J. Phys. A: Math. Theor. in press https://doi.org/10.1088/1751-8121/ab1441

\section{Manuscript version: Accepted Manuscript}

Accepted Manuscript is "the version of the article accepted for publication including all changes made as a result of the peer review process, and which may also include the addition to the article by IOP Publishing of a header, an article ID, a cover sheet and/or an 'Accepted Manuscript' watermark, but excluding any other editing, typesetting or other changes made by IOP Publishing and/or its licensors"

This Accepted Manuscript is @ 2018 IOP Publishing Ltd.

During the embargo period (the 12 month period from the publication of the Version of Record of this article), the Accepted Manuscript is fully protected by copyright and cannot be reused or reposted elsewhere.

As the Version of Record of this article is going to be / has been published on a subscription basis, this Accepted Manuscript is available for reuse under a CC BY-NC-ND 3.0 licence after the 12 month embargo period.

After the embargo period, everyone is permitted to use copy and redistribute this article for non-commercial purposes only, provided that they adhere to all the terms of the licence https://creativecommons.org/licences/by-nc-nd/3.0

Although reasonable endeavours have been taken to obtain all necessary permissions from third parties to include their copyrighted content within this article, their full citation and copyright line may not be present in this Accepted Manuscript version. Before using any content from this article, please refer to the Version of Record on IOPscience once published for full citation and copyright details, as permissions will likely be required. All third party content is fully copyright protected, unless specifically stated otherwise in the figure caption in the Version of Record.

View the article online for updates and enhancements. 
Keywords: non-equilibrium phase transitions, mathematical models for epidemics, random graphs, bifurcations, synergy

\title{
1. Introduction
}

Epidemics in networks is an important and interesting topic attracting a lot of research activity $[2,14,37,40]$. Recently, significant attention was paid to discontinuous phase transitions observed for spreading phenomena in complex networks $[3,6,7,9,13,20$ $22,28,32,35]$. Discontinuous phase transitions are typically exhibited by the socalled threshold models [37], such as the second Schlögl's model for autocatalytic reactions [41], quadratic contact process [27], the Watts model [33,46] and generalised contact process $[6,7]$. In these models, the nodes can be in different states which evolve according to certain dynamical rules. Within threshold models, the state of nodes changes when a certain threshold is achieved, e.g. a node can change its state when the number of its neighbours exceeds some threshold. Bi-stability regions for concentration

\begin{abstract}
S.N. Taraskin
Dept. of Chemistry, University of Cambridge, Cambridge CB2 1EW, UK

St. Catharine's College, University of Cambridge, Cambridge CB2 1RL, UK

E-mail: snt1000@cam.ac.uk

\section{F.J. Pérez-Reche}

Institute of Complex Systems and Mathematical Biology, SUPA, School of Natural and Computing Sciences, University of Aberdeen, Aberdeen AB24 3UE, UK

E-mail: fperez-reche@abdn.ac.uk

October 2018

Abstract. The role of cooperative effects (i.e. synergy) in transmission of infection is investigated analytically and numerically for epidemics following the rules of Sured thear dynamics are shown to lead to bifurcation diagrams for such spreading dependence of bifurcation loci on node degree is studied and interesting effects are found that contrast with the behaviour expected for non-synergistic epidemics.
\end{abstract}

\section{Bifurcations in synergistic epidemics on random regular graphs}




\section{Bifurcations in synergistic epidemics}

of infected nodes characteristic of discontinuous transitions were also found for an SIS model with adaptive network topology in which susceptible nodes try to avoid contacts with infected nodes by breaking the links with them and then rewiring these links to other randomly chosen nodes $[22,24,32]$. Bootstrap percolation on uncorrelated complex networks [3] provides one more example of discontinuous behaviour. This is a two-state model describing active and non-active nodes. A node becomes active and remains in such state if $k$ of its neighbours are active. The size of the giant active component can exhibit a discontinuous transition when the initial concentration of active nodes is used as a control parameter.

The synergy model introduced in Ref. [38] describes non-linear co-operative effects in communication between nearest neighbours in a network which can lead to discontinuous phase transitions in common epidemic models [21]. The key ingredient of the synergy model is that the transmission of infection is described by means of continuous (in contrast to discrete for the threshold models) functions of discrete variables such as the number of nearest neighbours in a certain state. Motivation for including synergy effects into the model comes from the experimental observations for e.g. soil-born epidemics [30] and spreading phenomena in social networks [12,26].

In this paper, we discuss the role of cooperative (synergistic) effects in transmission of infection and demonstrate how these effects can lead to discontinuous transitions. The analysis is undertaken for the SIS process spreading on random k-regular graphs. The aim of our analysis is two-fold and consists of developing a minimal and sufficient analytical framework (single-site mean-field) capturing all significant effects and suggesting a numerical procedure for supporting analytical findings. The description is presented in terms of bifurcation theory [44] which naturally suits our aims.

\section{The model}

\subsection{The rate equation}

We propose a model for synergistic SIS spread on a network of $N$ nodes whose connectivity defines a random $k$-regular graph in which each node is randomly connected to $k$ different nodes. The state of each node $i$ at time $t$ is characterised by a state variable $\sigma_{i}(t)$ which can be either $\sigma_{i}(t)=0$ or $\sigma_{i}(t)=1$ corresponding to susceptible $(\mathrm{S})$ or infected (I) state, respectively. In a time interval $\delta t$, nodes can change their state according to the following dynamical rules. If node $i$ is in the state I (i.e. $\sigma_{i}(t)=1$ ) at time step $t$ then it can change its state to $\mathrm{S}$ at time step $t+\delta t$ (i.e. $\sigma_{i}(t+\delta t)=0$ ) with probability $\mu \delta t$. Here, the parameter $\mu$ is the recovery rate which is assumed to be independent of the states of other nodes in the network and is the same for all nodes. If node $i$ is in the state $\mathrm{S}\left(\sigma_{i}(t)=0\right)$ then it can go to the state I at step $t+\delta t$ due to infection transferred from its $n_{i}(t)=\sum_{m=1}^{k} \sigma_{i_{m}}(t) \equiv \sum_{m=1}^{k} \sigma_{m}(t)$ infected neighbours where the node number $i_{m}$ of neighbour $m$ for brevity is replaced by just its nearest-neighbour index $m$. This occurs with probability $\Lambda_{n_{i}(t)} \delta t$, where $\Lambda_{n_{i}(t)}$ is the 
total transmission rate. The infection can be transmitted to node $i$ independently by any of its infected neighbouring nodes, $\left\{i_{j} \mid j=1, \ldots, n_{i}(t)\right\}$, with probability $\lambda_{i_{j}} \delta t$, where $\lambda_{i_{j}}$ is the individual transmission rate of infection from neigbouring infected node $i_{j}$ to node $i$. For brevity, the transmission rate will be denoted as $\lambda_{i j} \equiv \lambda_{i_{j}}$ in the following, i.e. the $j$-th infected neighbour of node $i$ is numbered by the index $j$. For independent transmissions in a particular configuration for node $i$ surrounded by $n_{i}(t)$ infected nodes, the total transmission rate is related to individual transmission rates as

$$
\Lambda_{n_{i}(t)} \delta t=1-\prod_{j}^{n_{i}(t)}\left(1-\lambda_{j i} \delta t\right) .
$$

In a standard formulation $[2,37]$ which ignores cooperative effects, the individual transmission rates are assumed to be constant values, $\lambda_{j i}=\lambda$, which do not depend on the state of neighbours of node $i$, so that the total transmission rate equals $\Lambda_{n_{i}(t)}=\left[1-(1-\lambda \delta t)^{n_{i}(t)}\right] / \delta t$. In case of the synergistic transmission, the individual transmission rates do depend on the neighbourhood of node $i$ and below we consider a case when individual transmission rates depend on the number of infected neighbours of node $i$, i.e. $\lambda_{j i}=\lambda_{n_{i}(t)}$, but do not depend on the properties of neighbour $j$ such as its degree $[21,38,45]$.

In order to make the definition (1) of the total transmission rate for synergistic transmission clearer, let us consider a simple example for a particular local configuration $C$ in a 3-regular graph. Assume that $C$ at time $t$ consists of a central uninfected node $i=0$, i.e. $\sigma_{0}(t)=0$, two infected neighbouring nodes $i_{1}=1(j=1)$ and $i_{2}=2(j=2)$, i.e. $\sigma_{1}(t)=1$ and $\sigma_{2}(t)=1$, and one uninfected neighbouring node $i_{3}=3$ with $\sigma_{3}(t)=0$, or symbolically $C=C_{2}=0_{0} \cap 1_{1} \cap 1_{2} \cap 0_{3}$ (the subscript in $C_{2}$ indicates that there are two infected neighbours). Let $T_{0}$ be the event that the central node changes its state at time $t+\delta t$ through transmission of infection from the infected neighbours. The infection can be transmitted to node 0 by means of several independent (by assumption) events, i.e. $T_{0}=\left(T_{10} \cap \bar{T}_{20}\right) \cup\left(T_{20} \cap \bar{T}_{10}\right) \cup\left(T_{10} \cap T_{20}\right)$, where $T_{j 0}\left(\bar{T}_{j 0}\right)$ is the event of transmission (nontransmission) of infection from infected neighbour $j$ to node 0 . Alternatively, infection might not be transmitted and this corresponds to the event $\bar{T}_{0}=\bar{T}_{10} \cap \bar{T}_{20}$. For a given configuration $C_{2}$, the probability for node 0 to be infected is $P\left(T_{0} \mid C_{2}\right)=1-P\left(\bar{T}_{0} \mid C_{2}\right)=$ $1-P\left(\bar{T}_{10} \cap \bar{T}_{20} \mid C_{2}\right)=1-P\left(\bar{T}_{10} \mid C_{2}\right) P\left(\bar{T}_{20} \mid C_{2}\right)=1-\left(1-P\left(T_{10} \mid C_{2}\right)\right)\left(1-P\left(T_{20} \mid C_{2}\right)\right)$, where $P\left(T_{j 0} \mid C_{2}\right)$ is the probability of transmission of infection from neighbour $j$ to node 0 given configuration $C_{2}$. This conditional probability $P\left(T_{j 0} \mid C_{2}\right)$ is defined in terms of individual transmission rates differently for synergistic and non-synergistic transmission. For non-synergistic transmission, $P\left(T_{j 0} \mid C_{2}\right)=P\left(T_{j 0} \mid 0_{0} \cap 1_{j}\right)=\lambda \delta t$, i.e. it is a non-zero constant value for all recipient-donor pairs in the network if recipient 0 is in S-state and donor $j$ is in I-state. In particular, the non-synergistic transmission probability $P\left(T_{j 0} \mid C_{2}\right)$ does not depend on the state of all other neighbours of node 0 except node $j$ and thus $P\left(T_{0} \mid C_{2}\right)=1-(1-\lambda \delta t)^{2}$. In contrast, for synergistic transmission, the value of $P\left(T_{j 0} \mid C_{2}\right)$ depends on the number of infected nodes in $C_{2}$, i,e. $P\left(T_{j 0} \mid C_{2}\right)=\lambda_{2} \delta t(j=1,2)$, and thus $P\left(T_{0} \mid C_{2}\right)=1-\left(1-\lambda_{2} \delta t\right)^{2}$ where index 2 


\section{Bifurcations in synergistic epidemics}

in the transmission rate refers to the two infected nodes in $C_{2}$. For configuration $C_{n}$ with $n$ infected neighbours surrounding a non-infected central node 0 , the synergistic transmission probability is $P\left(T_{0} \mid C_{n}\right) \equiv \Lambda_{n} \delta t=1-\left(1-\lambda_{n} \delta t\right)^{n}$ while the non-synergistic is $P\left(T_{0} \mid C_{n}\right) \equiv \Lambda_{n} \delta t=1-(1-\lambda \delta t)^{n}$. The two types of transmission are equivalent if $\lambda_{n}$ does not depend on number of infected neighbours of the recipient (susceptible central node), i.e. $\lambda_{n}=\lambda$.

Under these dynamical rules, the change of the probability $P\left(\sigma_{i}(t)=1\right) \equiv p_{i}(t)$ per unit time $\delta t$ obeys the following equation:

where

$$
\frac{\delta p_{i}(t)}{\delta t} \equiv \frac{p_{i}(t+\delta t)-p_{i}(t)}{\delta t}=R\left(p_{i},\left\{\sigma_{j}(t)\right\}\right)
$$

$$
R\left(p_{i},\left\{\sigma_{j}(t)\right\}\right)=-\mu p_{i}(t)+\sum_{n=1}^{k} \sum_{\left\{\sigma_{j}(t)\right\}} \Lambda_{n} P\left(\hat{0}_{i}(t),\left\{\sigma_{j}(t)\right\}\right) \delta_{n_{i}(t), n},
$$

is the rate function with $\Lambda_{n}$ obeying Eq. (1). The first contribution to $R$ is the probability for node $i$ to recover per time step $\delta t$, i.e. the recovery rate. The second contribution is the infection probability per unit time and it is proportional to the probability $P\left(0_{i}(t),\left\{\sigma_{j}(t)\right\}\right) \equiv P\left(\sigma_{i}(t)=0,\left\{\sigma_{j}(t)\right\}_{j=1}^{k}\right)$ for node $i$ to be in state $S$ and its neighbours in a configuration with states $\left\{\sigma_{j}(t)\right\}_{j=1}^{k}$ at time $t$. The total rate of infection is accounted for by summation over all the possible configurations of the neighbourhood, $\left\{\sigma_{j}(t)\right\}$, for all the possible values of the number of infected neighbours, $n$ (with $\delta_{n_{i}(t), n}$ being the Kronecker-delta).

For a particular example of the synergistic SIS process on a 3-regular graph, the double-summation term multiplied by $\delta t$ in Eq. (3) represents the probability of infection of susceptible node $i \equiv 0$ by its neighbours $(j=1,2,3)$ at time $t+\delta t$, i.e. $P\left(T_{0} \cap 0_{0}\right)=\sum_{\{C\}} P\left(T_{0} \mid C\right) P(C)$ where the summation is taken over configurations with fixed state of the central node, $\sigma_{0}(t)=0$, and all possible $\sigma_{j}(t)$ for its neighbours, i.e. explicitly,

$$
\begin{aligned}
& \delta t \sum_{n=1}^{3} \sum_{\left\{\sigma_{j}(t)\right\}} \Lambda_{n} P\left(0_{i}(t),\left\{\sigma_{j}(t)\right\}\right) \delta_{n_{i}(t), n}=\sum_{n=0}^{3} P\left(T_{0} \mid C_{n}\right) P\left(C_{n}\right) \\
& =\sum_{n=0}^{3}\left(1-\left(1-\lambda_{n} \delta t\right)^{n}\right) P\left(C_{n}\right),
\end{aligned}
$$

where $C_{n}$ is a configuration with $n$ infected neighbours of non-infected node 0 , i.e. $C_{0}=0_{0} \cap 0_{1} \cap 0_{2} \cap 0_{3}$ for no infected neighbours, $C_{1}=0_{0} \cap\left(\left(1_{1} \cap 0_{2} \cap 0_{3}\right) \cup\left(0_{1} \cap 1_{2} \cap 0_{3}\right) \cup\right.$ $\left.\left(0_{1} \cap 0_{2} \cap 1_{3}\right)\right)$ for one infected neighbour, $C_{2}=0_{0} \cap\left(\left(1_{1} \cap 1_{2} \cap 0_{3}\right) \cup\left(1_{1} \cap 0_{2} \cap 1_{3}\right) \cup\left(0_{1} \cap 1_{2} \cap 1_{3}\right)\right)$ for two infected neighbours, and $C_{3}=0_{0} \cap 1_{1} \cap 1_{2} \cap 1_{3}$ for three infected neighbours. The probabilities of configurations with particular number of infected nodes are given by standard expressions, e.g. $P\left(C_{2}\right)=P\left(0_{0} \cap 1_{1} \cap 1_{2} \cap 0_{3}\right)+P\left(0_{0} \cap 1_{1} \cap 0_{2} \cap 1_{3}\right)+P\left(0_{0} \cap\right.$ $\left.0_{1} \cap 1_{2} \cap 1_{3}\right)$.

Eq. (3) for marginal probabilities $p_{i}$ can be obtained from the full set of $2^{N}$ rate equations for the state probabilities $P\left(\left\{\sigma_{i}(t)\right\}_{i=1}^{N}\right)$, which are similar to Eq. (2), by 
summing up all the equations with fixed value of $\sigma_{i}(t)=1$ on the left-hand side of these equations. The sum taken over all state variables equals zero, reflecting the conservation of probability while the partial sum with fixed $\sigma_{i}(t)=1$ leads to a lot of cancellations for the rate terms (on the right-hand side of these equations), describing transition events occurring away from the neighbourhood of node $i$ so that the surviving terms describe only the events in which the nearest neighbours of $i$ are involved. The rate equations similar to Eq. (3) but without synergy effects are used for description of spreading processes, especially in dealing with dynamical correlations $[17,39,43]$.

The model introduced above is well defined for any $\delta t<1 / \lambda_{n}$ but we will focus on two dynamics:

(i) Discrete-time dynamics (d-time): The state of nodes changes in discrete time steps, $\delta t$, and they do so simultaneously (i.e. updates are synchronous). We set $\delta t=1$ for d-time dynamics in the sequel which implies that the rates coincide with probabilities.

(ii) Continuous time dynamics (c-time): The time step is infinitesimal, i.e. $\delta t \rightarrow d t$. Accordingly, $\delta p_{i} \rightarrow d p_{i}$ and $\Lambda_{n}=n \lambda_{n}$

\subsection{Forms of synergy}

In our model, the synergistic effects are incorporated in the individual transmission rates, $\lambda_{n}$. Two particular synergistic mechanisms are analysed below: S-synergy and I-synergy.

For S-synergy, the $k-n_{i}$ susceptible neighbours of node $i$ multiplicatively affect the individual transmission rates. In general, this can be represented by an exponential functional form, i.e.

$$
\lambda_{n_{i}}=\min \left\{\alpha e^{\beta\left(k-n_{i}\right)}, 1 / \delta t\right\},
$$

with $\alpha$ being the inherent transmission rate when all the neighbours of $i$ are infected $\left(n_{i}=k\right)$ and there are no susceptible neighbours of $i$ which could affect the strength of the attack by the infected neighbours. The parameter $\beta$ controls the synergy strength of susceptible neighbours. Positive values of $\beta$ correspond to constructive synergy in which the susceptible neighbours of $i$ encourage the transmission. In contrast, negative values represent situations in which susceptible neighbours of $i$ multiplicatively cooperate to prevent transmission to $i$. For example, the transmission towards a node $i$ of degree $k>1$ is reduced by a factor $e^{\beta}$ when there is only one susceptible node connected to $i$. Similarly, if two neighbours of node $i$ are in susceptible state, the greater support leads to the reduction of infection rate by factor $\left(e^{\beta}\right)^{2}$, etc. The minimum condition in Eq. (5) ensures that the probability of transmission in time step $\delta t$ is at most 1 for any $\alpha$ and $\beta$. This form of synergy was proposed as an important factor for the spread of social content [21]. Other functional forms of $\lambda_{n}$ can be used as well although the main features of the synergistic SIS processes are expected to be qualitatively similar (see e.g. $[21,38,45]$ where a linear dependence of $\lambda_{n}$ on $n$ corresponding to additive cooperation was studied). 


\section{Bifurcations in synergistic epidemics}

For the second type of synergy, I-synergy, the individual transmission rates are multiplicatively affected by $n_{i}$ infected neighbours of susceptible node $i$ which the infection is attempted to be passed to [38], i.e.

$$
\lambda_{n_{i}}=\min \left\{\alpha e^{\beta\left(n_{i}-1\right)}, 1 / \delta t\right\} .
$$

Here, $\alpha$ is the inherent rate of infection corresponding to the case in which transmission to $i$ comes from a single infected neighbour, i.e. when $n_{i}=1$. The synergy parameter $\beta$ accounts for the strength of cooperation $(\beta>0)$ or interference $(\beta<0)$ between the infected neighbours of $i$.

It follows from comparison of Eqs. (5) and (6) that I-synergy is similar to Ssynergy with inverse sign of the synergy strength, i.e. with $\beta$ replaced by $-\beta$ in Eq. (5) although with a significant distinction. Indeed, Eq. (5) can be rewritten as $\lambda_{n_{i}}=\left(\alpha e^{-\beta(1-k)}\right) e^{-\beta\left(n_{i}-1\right)}$ so that it has the form of Eq. (6) but with inherent transmission rate $\left(\alpha e^{-\beta(1-k)}\right)$ dependent on the node degree $k$. In contrast, the inherent transmission rate for I-synergy in Eq. (6) is the same for all nodes independent of their degree. This difference between two types of synergy can be especially significant in heterogeneous networks where the nodes have different degrees.

For both types of synergy, the individual transmission rates are continuous functions of the discrete variable $n_{i}$ giving the number of infected neighbours. This is in contrast to the threshold models where the transmission rates are described by discontinuous (threshold) functions of $n_{i}[6,7,9,27,35,43,47]$. The synergistic individual transmission rates are determined by two parameters, i.e. by the inherent transmission rate $\alpha$ and by the synergy strength $\beta$, and their dependence on $\alpha$ and $\beta$ is described by continuous functions. In the synergy-free case, i.e. for $\beta=0$, these rates do not depend on $n_{i}$, and just coincide with the inherent transmission rate. All these properties of the synergistic transmission rates make possible to investigate the influence of cooperative effects on SIS process in the whole $(\beta, \alpha)$ parameter space and reveal, as shown below, quite a rich behaviour even for the simplest case of the SIS spread on k-regular graphs.

\section{Methods}

To analyse the proposed model, one can either simulate the process numerically by means of Monte Carlo (MC) sampling of the trajectories of the system or through analytical approaches after making certain approximations [37]. Both procedures were employed to analyse the model. Before presenting the results of our analysis, we describe the ingredients of our analytical calculations.

The analytical results are based on two standard approximations [20,37]: the singlesite approximation and mean-field approximation (sometimes they both are called just a mean-field approximation [19]). The single-site approximation neglects dynamical correlations, i.e. $P\left(0_{i},\left\{\sigma_{j}(t)\right\}\right)=\left(1-p_{i}\right) \prod_{j=1}^{k_{i}} \pi\left(\sigma_{j}(t)\right)$, where $\pi\left(\sigma_{j}(t)=1\right)=p_{j}$ and $\pi\left(\sigma_{j}(t)=0\right)=1-p_{j}$

The homogeneous mean-field approximation assumes that the probability of a node being infected does not depend on the node, i.e. $p_{i}=p$ for all $i$. Despite the fact 


\section{Bifurcations in synergistic epidemics}

that this approximation neglects fluctuations completely, it is reasonable because, in the thermodynamic limit, random k-regular graphs do not contain loops and in finite networks the number of such loops is exponentially small [8]. Combining these two approximations, the rate function (see Eq. (3)) becomes:

$$
R\left(p_{i},\left\{\sigma_{j}(t)\right\}\right) \simeq R(p) \equiv-\mu p+(1-p) q(p)
$$

where

$$
q(p)=\sum_{n=1}^{k}\left(\begin{array}{l}
k \\
n
\end{array}\right) \Lambda_{n} p^{n}(1-p)^{k-n}
$$

is the rate at which a susceptible individual gets infected by its infected neighbours.

A closed form can be found for $q(p)$ for both I- and S-synergy in the c-time limit:

$$
q(p)= \begin{cases}\alpha k p\left[(1-p) e^{\beta}+p\right]^{k-1}, & \text { for S-synergy } \\ \alpha k p\left[1-p+p e^{\beta}\right]^{k-1}, & \text { for I-synergy }\end{cases}
$$

Note that the heuristic expression $q(p)=\alpha k p e^{\beta k(1-p)}$ proposed in [21] for d-time S-synergy differs from the expressions derived here more rigorously for both c- and d-time dynamics. The expression given in [21] coincides with those given here for nonsynergistic spread $(\beta=0)$ and captures the key trends when $\beta \neq 0$. For instance, it is a monotonically increasing function of $p$ for any $\alpha, k>0$. Strictly speaking, however, one should use Eq. (8) in order to describe accurately the synergistic effects of any transmission rate within the single-site mean-field approximation.

\section{Results}

The behaviour of the process in the long-time limit $t \rightarrow \infty$ is of special interest because it determines if the network is vulnerable to the infection spread or not. We will analyse the effect of synergistic transmission in this limit in which the SIS process reaches a stationary (quasi-stationary in finite systems) regime such that $R\left(p_{i},\left\{\sigma_{j}(t)\right\}\right)=0$.

Due to the non-linear nature of the rate function, the number of its fixed points can vary when the parameters of the model change and different stationary regimes can emerge in the parameter space. For c-time dynamics and given node degree, stationary states can be fully parametrised with two parameters, $\tilde{\alpha}=\alpha / \mu$ and $\beta$ since $R(p)$ is linear in $\lambda_{n}$ (this is clear from Eq. (3) and expression $\Lambda_{n}=n \lambda_{n}$ for c-time). In contrast, the stationary states for d-time processes depend on $\alpha, \beta$ and $\mu$. This is an important difference compared to the stationary states for non-synergistic SIS processes that depend on $\tilde{\alpha}$ for c-time and $\tilde{\alpha}$ and $\mu$ for d-time dynamics.

Different stationary regimes are separated in the parameter space by bifurcations at which the number of stationary points (roots of $R(p)$ ) changes [44]. The results presented below involve bifurcations of three different types:

(i) Transcritical (TC) bifurcation. This is a codimension-one bifurcation point and it corresponds to double root at $p_{0}=0$ with condition, $R(0)=R^{\prime}(0)=0$. The 
Bifurcations in synergistic epidemics

infection probability $p$ changes continuously when a TC bifurcation is crossed in the parameter space.

(ii) Saddle-node (SN) bifurcation. This is a codimension-one bifurcation point and it corresponds to double root at finite density, $p_{*}$, with conditions, $R\left(p_{*}\right)=R^{\prime}\left(p_{*}\right)=0$, for $p_{*} \in(0,1)$. The stationary density changes discontinuously when a SN point is crossed.

(iii) Saddle-node-transcritical (SNT) crossing bifurcation. This is a codimension-two bifurcation point and it corresponds to triple root at zero density with conditions, $R(0)=R^{\prime}(0)=R^{\prime \prime}(0)=0$. The stationary density near zero changes continuously when an SNT point is crossed.

\subsection{S-synergy}

The location of the bifurcation points in $(\beta, \tilde{\alpha})$ parameter space,i.e. bifurcation diagram, for SIS process exhibiting S-synergy in transmission on random k-regular graph is shown in Fig. 1(a) for a typical set of parameters. The data presented by lines were obtained withing the single-site mean-field approximation while the open symbols correspond to results of MC simulations (see Sec. 5 for more detail). There are three different regimes: regime I (non-active), regime II (active) and regime III (bi-stable). A general analysis of stability of all fixed points found in the paper is given in Appendix A. In regime I, the synergistic SIS process is characterised by a single fixed point located at $p_{0}=0$ which is globally asymptotically stable in the feasible interval with $p \in(0,1$ ] (see Corollary Appendix A.1.2). The solid line in Fig. 1(c) shows an example of the rate function $R(p)$ which only has a root at $p=p_{0}=0$. In regime II, there are two fixed points located at $p_{0}=0$ and $p_{1}>0$ (see the dot-dashed $R(p)$ curve in Fig. $1(\mathrm{c})$ ). The fixed point at $p_{0}$ is unstable and processes in this regime evolve towards the active regime with fixed point at $p_{1}$ which is globally asymptotically stable in the feasible interval (see Corollary Appendix A.1.3). Regime III is characterised by three fixed points, two locally stable at $p_{0}=0$ and $p_{2}>0$ and one unstable at $p_{1} \in\left(p_{0}, p_{2}\right)$ (see the dashed curve in Fig. 1(c) and Corollary Appendix A.1.4). Note that the bifurcation (phase) diagram of the model is qualitatively similar to that presented in [21] but there are quantitative differences due to different treatment of the function $q(p)$.

The different regimes are separated by lines of bifurcations. The solid line in Fig. 1(a), $\tilde{\alpha}=\tilde{\alpha}_{\mathrm{TC}}(\beta)$, represents the TC bifurcations separating active and non-active regimes on the right $\left(\beta>\beta_{\mathrm{SNT}}\right)$ to the $\mathrm{SNT}$ crossing bifurcation (solid square) at $\left(\beta_{\mathrm{SNT}}, \tilde{\alpha}_{\mathrm{SNT}}\right)$, and active and bi-stable regimes on the left $\left(\beta<\beta_{\mathrm{SNT}}\right)$ to the SNT point, respectively. The broken style lines with $\tilde{\alpha}=\tilde{\alpha}_{\mathrm{SN}}(\beta)$ correspond to the SN bifurcations between non-active and bi-stable regimes.

For relatively large fixed values of $\beta>\beta_{\mathrm{SNT}}$, the SIS process is in non-active regime I for $\tilde{\alpha}<\tilde{\alpha}_{\mathrm{TC}}$ and in active regime II for $\tilde{\alpha}>\tilde{\alpha}_{\mathrm{TC}}$. In this case, the probability $p$ for a node to be infected or equivalently the concentration of infected nodes is a single-valued function of $\tilde{\alpha}$ and $\beta$ (see the dashed and dot-dashed curves in Fig. 1(b)). Within the 

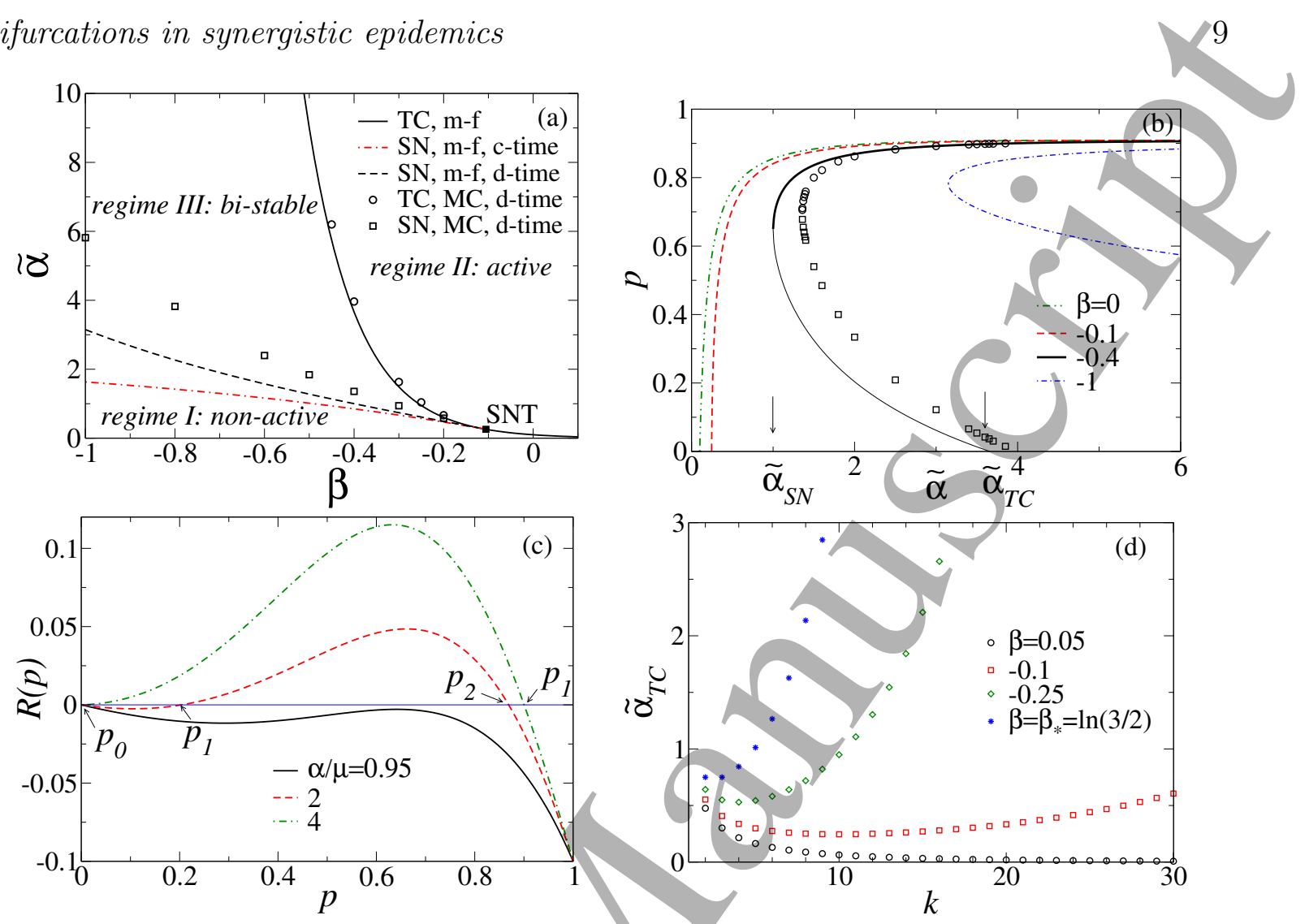

Figure 1. (a) Bifurcation diagram for SIS processes with S-synergy on a random kregular graph with $k=10$ following c- and d-time dynamics. The solid line represents the line of TC biburcations given by mean-field (m-f) Eq. (10). The dashed and dot-dashed lines show SN bifurcations for d-time dynamics with $\mu=0.1$ and c-time dynamics (see Eq. (13)), respectively. The circles (TC biburcations) and squares (SN bifurcations) represent the data obtained numerically for random 10-regular graphs of size $N=10^{5}$ for SIS processes following the rules of d-time dynamics with $\mu=0.1$. The error bars for MC data are lees than the symbols size. The solid square shows the SNT crossing bifurcation with coordinates given by Eqs. (11)-(12) for c-time dynamics. (b) Dependence of the concentration of infected nodes $p$ in quasi-equilibrium state for SIS process with S-synergy on random 10-regular graph $\left(N=10^{5}\right)$ vs relative transmission probability $\tilde{\alpha}$ for d-time dynamics with $\mu=0.1$ and different values of the synergy parameter $\beta$ shown in the legend. In the bi-stable regime for $\beta=-0.4$, the thick and thin solid lines correspond to finite-density stable and unstable stationary states of the synergistic SIS process, respectively. Squares (unstable equilibrium) and circles (stable equilibrium) show numerical results for d-time dynamics with $\mu=0.1$ and $\beta=-0.4$. (c) Dependence of the rate function, $R(p)$, calculated within the singlesite mean-field approximation on the concentration of infected nodes, $p$, for d-time SIS process with $\mu=0.1, \beta=-0.4$ and different values of $\tilde{\alpha}=\alpha / \mu$ as indicated in the legend. (d) Dependence of the TC bifurcation point, $\tilde{\alpha}_{\mathrm{TC}}(\beta ; k)$ given by Eq. (10), on the node degree $k$ for random k-regular graphs. 

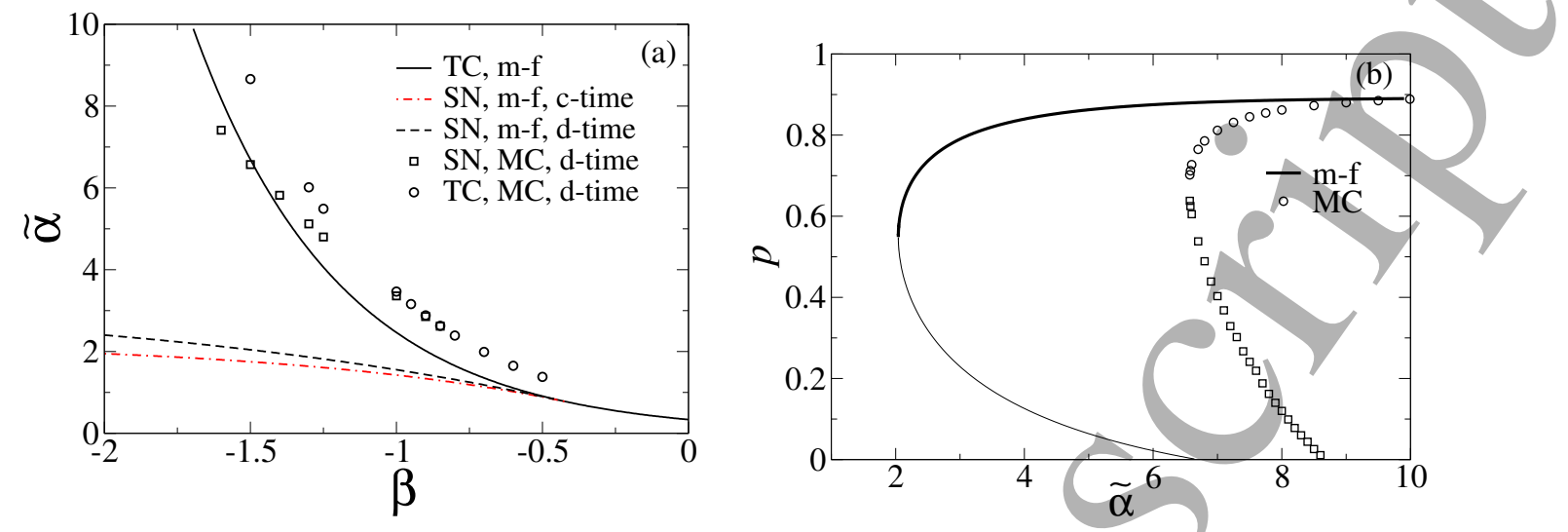

Figure 2. (a) Bifurcation diagram for SIS processes with S-synergy on a random 3 -regular graph. The same values of parameters and line and symbol styles as in Fig. 1(a) are used. (b) Dependence of the concentration of infected nodes $p$ in quasiequilibrium state for an SIS process with S-synergy on a random 3-regular graph vs relative transmission probability $\tilde{\alpha}$ for d-time dynamics with $\beta=-1.5$. The meanfield $(\mathrm{m}-\mathrm{f})$ and MC simulations data are shown by lines and symbols, respectively. The values of other parameters used in the simulations are the same as those for data shown in Fig. 1(b).

single-site mean-field approximation, the functional form for the TC bifurcation line and coordinates of the SNT crossing bifurcation can be found analytically both for d- and c-time dynamics. Indeed, the TC bifurcation line where $R^{\prime}(0)=0$ satisfies

$$
\tilde{\alpha}_{\mathrm{TC}}(\beta)=\frac{1}{k} e^{-\beta(k-1)}
$$

for both $\mathrm{d}$ - and c-time dynamics. This line is independent of $\mu$ and reduces to the wellknown threshold condition $\tilde{\alpha}_{\mathrm{TC}}(\beta)=1 / k$ for non-synergistic epidemics with $\beta=0[2,37]$.

The coordinates of the SNT crossing bifurcation point (solid square in Fig. 1(a)), where $R(0)=R^{\prime}(0)=R^{\prime \prime}(0)$, are given by the following expressions,

$$
\begin{array}{ll}
\tilde{\alpha}_{\mathrm{SNT}}= \begin{cases}\frac{1}{k}\left[\frac{k}{\mu}\left(1-\sqrt{1-\frac{2 \mu}{k-1}}\right)\right]^{k-1}, & \text { for d-time } \\
\frac{1}{k}\left(\frac{k}{k-1}\right)^{k-1}, & \text { for c-time }\end{cases} \\
\beta_{\mathrm{SNT}}= \begin{cases}-\ln \left[\frac{k}{\mu}\left(1-\sqrt{1-\frac{2 \mu}{k-1}}\right)\right], & \text { for d-time } \\
\ln \left(\frac{k-1}{k}\right), & \text { for c-time. }\end{cases}
\end{array}
$$

Note that the coordinates of the SNT crossing bifurcation for c-time dynamics are obtained by the limit for $\mu \rightarrow 0$ in the expressions for d-time dynamics.

For sufficiently negative fixed values of $\beta<\beta_{\mathrm{SNT}}$ (to the left of the SNT crossing bifurcation point in Fig. 1(a)), the behaviour of the synergistic SIS processes changes drastically. In particular, $p$ becomes a multivalued function of $\tilde{\alpha}$ in the interval 
$\left(\tilde{\alpha}_{\mathrm{SNT}}(\beta), \tilde{\alpha}_{\mathrm{TC}}\right)$ where the SIS process is in bi-stable regime III (see the multi-valued function $p(\tilde{\alpha})$ shown by the solid line in Fig. 1(b)). In the bi-stable regime, the equilibrium concentration of infected nodes depends on initial concentration of infected nodes, $p(0)$. Within mean-field, the SIS process is non-active if $p(0)<p_{1}$, where $p_{1}$ is the middle root of $R(p)$. In contrast, it is active if $p_{1}<p(0)$, i.e. it reaches a finite concentration $p=p_{2}>0$, where $p_{2}$ is the largest root of $R(p)$.

The expression for $\tilde{\alpha}_{\mathrm{SN}}(\beta)$ corresponding to the line of SN bifurcations can be found analytically only for c-time dynamics (the result for d-time dynamics can be obtained numerically). Indeed, using the condition $R\left(p_{*}\right)=R^{\prime}\left(p_{*}\right)=0$ and Eq. (9) for c-time S-synergy, one obtains

$$
\tilde{\alpha}_{\mathrm{SN}}(\beta)=\left(\frac{k}{k-1}\right)^{k-1}\left(1-e^{\beta}\right)
$$

which is valid for $\beta \leq \beta_{\mathrm{SNT}}$. For d-time dynamics, the shape of the SN bifurcation line depends on the value of $\mu$ and tends to the SN bifurcation line for c-time dynamics (the dot-dashed line in Fig. 1(a)) when $\mu \rightarrow 0$.

We analysed above the behaviour of SIS processes in a representative case of random $\mathrm{k}$-regular graphs with $k=10$. However, all the qualitative findings hold for other values of $k$ as well. As an example, in Fig. 2, we show the bifurcation diagram and dependence of concentration of infected nodes on the reduced transmission rate, $\tilde{\alpha}$, for 3-regular graph. As seen from comparison of Fig. 2 with Fig. 1, qualitatively the behaviour of the synergistic SIS process is the same in both 3- and 10-regular graphs. However, the quality of the single-site mean-field approximation in description of the synergistic SIS processes studied numerically becomes noticeably better with increasing $k$. This is a known effect according to which the states of two neighbouring nodes are less likely to depend on the state of each other if they are connected to many other neighbours which are more likely to influence the pair of neighbours $[19,20]$.

The effect of the node degree on different regimes exhibited by the model can be readily analysed using the analytical expressions for bifurcations. We restrict our analysis to $k \geq 2$ to ensure a giant connected component in the network. The region corresponding to regime III in the $(\beta, \tilde{\alpha})$ space increases with $k$, i.e. bi-stable behaviour is overall more likely when increasing the connectivity of the network. Indeed, for $\beta<\beta_{\mathrm{SNT}}$, the relative inherent transmission rate $\tilde{\alpha}_{\mathrm{SN}}(\beta ; k)$ exhibits a mild increase with $k$ that is counteracted by a faster increase of $\tilde{\alpha}_{\mathrm{TC}}(\beta ; k)$. This results in an enlargement of the region for bi-stable behaviour with increasing $k$.

The dependence of $\tilde{\alpha}_{\mathrm{TC}}(\beta ; k)$ on $k$ is more interesting. Depending on the value of $\beta$, one can distinguish three cases (see Fig. 1(d)).

(i) $\beta<\beta_{*} \equiv \ln (2 / 3)$ : One can prove (bearing in mind that $\tilde{\alpha}_{\mathrm{TC}}\left(\beta_{*} ; k=2\right)=$ $\tilde{\alpha}_{\mathrm{TC}}\left(\beta_{*} ; k=3\right)$; see the stars in Fig. $\left.1(\mathrm{~d})\right)$ that $\tilde{\alpha}_{\mathrm{TC}}(\beta ; k)$ increases monotonically with $k \geq 2$. This behaviour can be intuitively understood in terms of a higher opposition of susceptible neighbours to transmission for large $k$. 


\section{Bifurcations in synergistic epidemics}

(ii) $\beta_{*}<\beta<0$ : This is a more counter-intuitive regime since $\tilde{\alpha}_{\mathrm{TC}}(\beta ; k)$ has a minimum at $k=\left\lceil e^{\beta}\left(1-e^{\beta}\right)^{-1}\right\rceil$, where $\lceil\cdot\rceil$ is the ceiling function (see the squares and diamonds in Fig. 1(d)). This means that there is a value of $k$ for which the system is particularly vulnerable to leaving the non-active regime.

(iii) $\beta>0$ : The intrinsic rate $\tilde{\alpha}_{\mathrm{TC}}(\beta)$ decreases monotonically with $\beta$ (see the circles in Fig. 1(d)) . This behaviour is again intuitively expected since susceptible neighbours encourage transmission from infected nodes and this makes the invasion more likely (i.e. occurs for smaller values of $\alpha$ ) if connectivity is large. Transitions in this case are always between regimes I and II.

\subsection{I-synergy}

Fig. 3(a) shows a typical phase diagram for SIS processes exhibiting I-synergy in transmission and spreading on random k-regular graphs with $k=10$. As in the case of S-synergy, there are three different regimes: regime I'non-active), regime II (active) and regime III (bi-stable). As follows from this figure, only constructive I-synergy affects the SIS processes making them more invasive as compared to the synergy-free case. Indeed, if $\beta>\beta_{\mathrm{SNT}}$ then even for relatively small inherent infection rates, i.e. $\tilde{\alpha}<\tilde{\alpha}_{\mathrm{TC}}$ (but $\left.\tilde{\alpha}>\tilde{\alpha}_{\mathrm{SN}}\right)$ the network becomes vulnerable for invasion and the concentration of infected nodes can increase abruptly from zero to a finite value (see the curves in Fig. 3(b) for $\beta>\beta_{\mathrm{SNT}} \simeq 0.11096$ ). The location of bifurcation points can be found within the single-site mean-field approximation following similar steps as above for S-synergy. A TC bifurcation occurs at an inherent rate given by

$$
\tilde{\alpha}_{\mathrm{TC}}(\beta)=\frac{1}{k}
$$

which is not affected by synergy (does not depend on $\beta$; see the horizontal line in Fig. 3(a)) and coincides with that for the synergy-free case [2,37]. This is because it is determined by transmission events with a single attacker only. Despite not affecting $\tilde{\alpha}_{\mathrm{TC}}(\beta)$, constructive I-synergy can affect the SIS processes so that they become bistable (see Fig. 3(a)) in a similar way to the SIS processes with S-synergy. A single SNT crossing bifurcation is present in the bifurcation diagram and its coordinates can be obtained analytically for both c- and d-time dynamics:

$$
\begin{array}{ll}
\tilde{\alpha}_{\mathrm{SNT}}=\frac{1}{k} & \text { for both c- and d-time } \\
\beta_{\mathrm{SNT}}= \begin{cases}\ln \left[\frac{k}{\mu}\left(1-\sqrt{1-\frac{2 \mu}{k-1}}\right)\right], & \text { for d-time } \\
\ln \left(\frac{k}{k-1}\right), & \text { for c-time }\end{cases}
\end{array}
$$

The expressions for both the value of the elementary rate, $\tilde{\alpha}_{\mathrm{SN}}(\beta)$, and concentration of infected nodes, $p_{\mathrm{SN}}(\beta)$, can be obtained analytically for c-time dynamics at the SN 
Bifurcations in synergistic epidemics

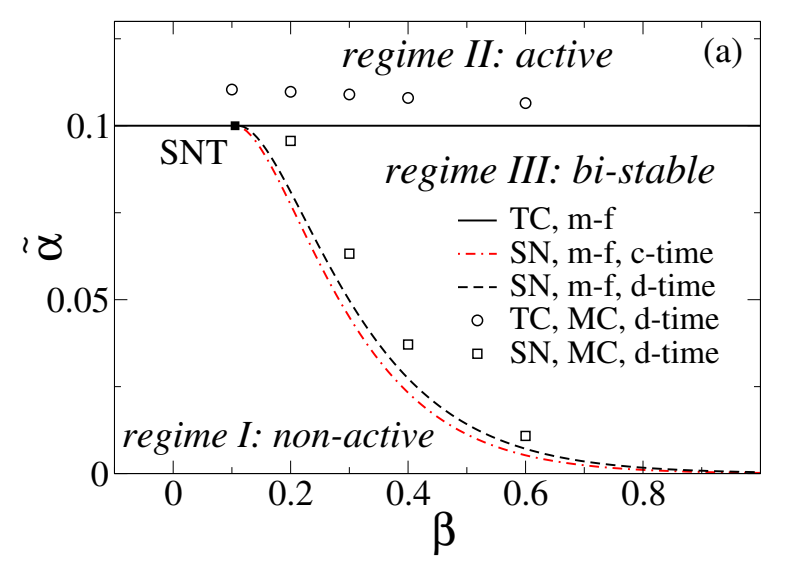

Figure 3. (a) Bifurcation diagram for SIS processes with I-synergy on random kregular graphs with $k=10$ following c- and d-time dynamics. The solid line represents the line of TC biburcations given by Eq. (14). The dashed and dot-dashed lines show SN bifurcations for d-time dynamics with $\mu=0,1$ and c-time dynamics (see Eq. (17)), respectively. The circles (TC biburcations) and squares (SN bifurcations) represent the data obtained numerically for random 10-regular graphs of size $N=10^{5}$ for SIS processes following the rules of d-time dynamics with $\mu=0.1$. The solid square shows the SNT crossing bifurcation with coordinates given by Eqs. (15)-(16) for c-time dynamics. (b) Dependence of the concentration of infected nodes $p$ in quasi-equilibrium state for SIS process with I-synergy on random 10-regular graph $\left(N=10^{5}\right)$ vs relative transmission probability $\tilde{\alpha}$ for d-time dynamics with $\mu=0.1$ and different values of the synergy parameter $\beta$ shown in the legend. In bi-stable regime for $\beta=-0.4$, the thick and thin solid lines correspond to finite-density stable and unstable stationary states of the synergistic SIS process, respectively. Squares (unstable stationary state) show numerical results for d-time dynamics $\mu=0.1$ and $\beta=-0.4$.

bifurcation separating non-active and bi-stable regimes for $\beta \geq \beta_{\mathrm{SNT}}$ :

$$
\begin{aligned}
& \tilde{\alpha}_{\mathrm{SN}}(\beta)=\left(\frac{k}{k \jmath^{1}}\right)^{k-1}\left(e^{\beta}-1\right) e^{-\beta k}, \\
& p_{\mathrm{SN}}(\beta)=1-\frac{1}{k\left(1-e^{-\beta}\right)} .
\end{aligned}
$$

In case of d-time dynamics, these quantities can be calculated numerically.

\subsection{Invasion threshold}

The existence of a well-defined threshold separating non-active and active regimes is an appealing idea in mathematical epidemiology which is often expressed in terms of the basic reproduction number $R_{0}[25,37]$. A well-defined threshold exists under quite general conditions [15] and corresponds to a TC bifurcation separating regimes I and II with $R_{0}=1$ at the threshold. Here, we show that synergistic effects restrict the regime of validity of the concept of a threshold defined by condition $R_{0}=1$. Similar deviations

from the normal threshold criterion were found in previous works studying the effect of non-linear incidence rates on invasions $[23,29]$. 


\section{Bifurcations in synergistic epidemics}

For the models studied here, one can define a basic reproduction number as

$$
R_{0}=\frac{k}{\mu} \lambda_{1}
$$

This formula can be intuitively interpreted as the average number of nodes that become infected at the initial stages of the epidemic by the transmission of infection with rate $\lambda_{1}$ from an infected node during its infectious period which is of the order of $1 / \mu$.

By using $\lambda_{1}=\alpha e^{\beta(k-1)}$ for S-synergy and condition (10) for the TC bifurcation, one can express the reproductive number as $R_{0}=\alpha / \alpha_{\mathrm{TC}}$. Therefore, the condition for the TC bifurcation along the line $\alpha_{\mathrm{TC}}(\beta)$ is clearly equivalent to the common threshold condition $R_{0}=1$. The threshold concept defined by $R_{0}=1$ is, however, only meaningful for $\beta \geq \beta_{\mathrm{SNT}}$. Indeed, for $\beta<\beta_{\mathrm{SNT}}$, the active regime is already stable in regime III where $\alpha \in\left(\alpha_{\mathrm{SN}}(\beta), \alpha_{\mathrm{TC}}(\beta)\right)$. This implies that, depending on the initial conditions, synergistic invasions are possible for $R_{0}>\alpha_{\mathrm{SN}}(\beta) / \alpha_{\mathrm{TC}}(\beta)$, i.e. they are possible even if $R_{0}<1$.

The reproduction number in case of I-synergy can/be similarly expressed as $R_{0}=\alpha / \alpha_{\mathrm{TC}}$ but with $\alpha_{\mathrm{TC}}=\mu / k$ (cf. Eq. (14)). Following a similar reasoning as for S-synergy it is easy to demonstrate that the definition of the threshold by condition $R_{0}=1$ is only meaningfull for $\beta \leq \beta_{\mathrm{SNT}}$. For $\beta>\beta_{\mathrm{SNT}}$, invasions are again possible in regime III where $R_{0}<1$.

\subsection{A minimal model}

In the presence of synergy, the rate function $R(p)$ given by Eq. (8) is a polynomial of order $k$. However, we have only found regimes with at most three roots for $R(p)$. This suggests the existence of a simplified normal form for the model which can capture all the three dynamical regimes described above. Indeed, a cubic normal form, $R_{\mathrm{nf}}$, for the rate function,

$$
R_{\mathrm{nf}}(p)=a p+b p^{2}-c p^{3}
$$

is sufficient to qualitatively capture all the regimes predicted by the full model for any $k$. A similar normal form was proposed in [42] to describe the interaction of TC and SN bifurcations in an extended Lotka-Volterra model.

The normal form is obtained through an expansion of $R(p)$ given by Eq. (8) up to and including terms $\propto \mathrm{O}\left(\mathrm{p}^{3}\right)$ around $p=0$ which gives the following expressions for coefficients in Eq. (20):

$$
\begin{aligned}
& a=-\mu+k \Lambda_{1}, \\
& 2 b=k(k-1) \Lambda_{2}-2 k^{2} \Lambda_{1}, \\
& 6 c=-k(k-1)\left[(k-2) \Lambda_{3}-3(k-1) \Lambda_{2}+3 k \Lambda_{1}\right] .
\end{aligned}
$$

Since $R(1)<0$, a necessary condition for the normal form $R_{\mathrm{nf}}(p)$ to capture this behaviour is that $c>0$, so that $R_{\mathrm{nf}}(p=1 ; a=b=0)<0$. If $c>0$ and the parameters $a$ and $b$ vary, the shape of $R_{\mathrm{nf}}(p)$ evolves in a similar way to that of $R(p)$ 

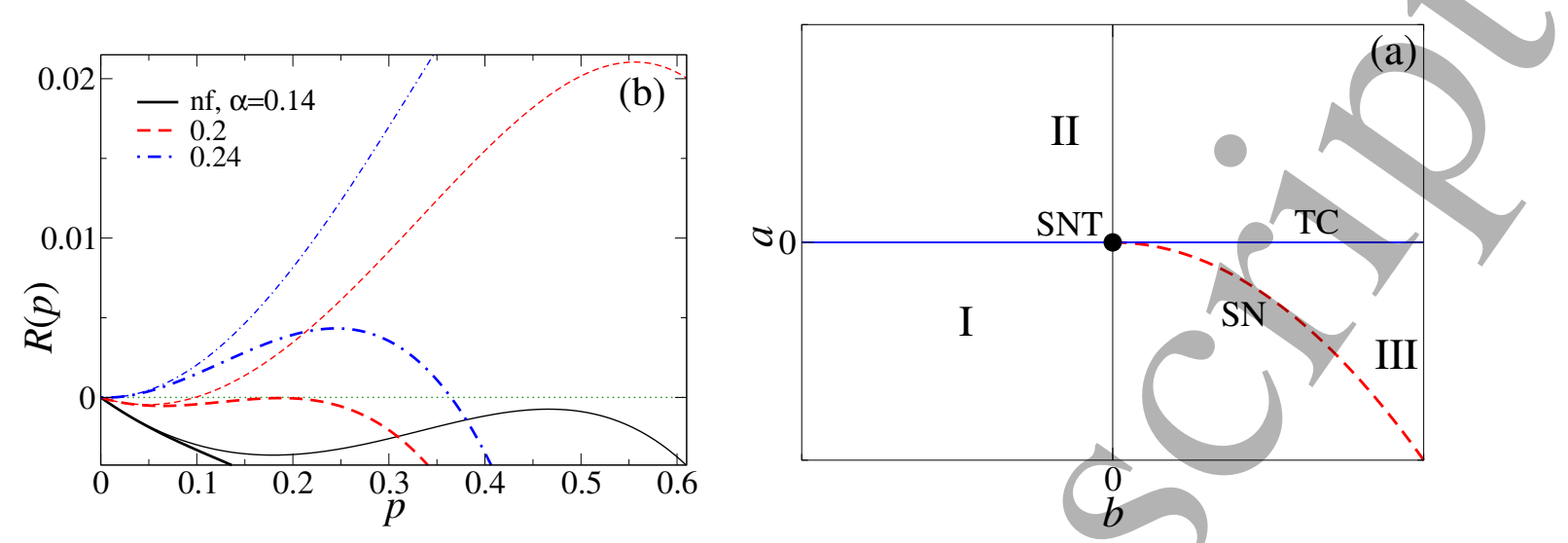

Figure 4. (a) Comparison of the normal form $R_{\mathrm{nf}}(p)$ (bold lines, Eq. (20)) and meanfield rate function $R(p)$ (thin lines) for $k=3, \mu=0.1, \beta=-1<\beta_{\mathrm{SNT}} \simeq-0.43$ and several values of $\alpha$ as indicated in the legend. (b) Bifurcation diagram for the minimal model based on the normal form for the rate function, $R_{\mathrm{nf}}(p)$.

when parameters $\alpha$ and $\beta$ vary. This is illustrated by Fig, 4(b) which shows an example in which both $R(p)$ and $R_{\mathrm{nf}}(q)$ (cf. thin and bold lines) exhibit the expected shapes for $\beta<\beta_{\mathrm{SNT}}$, i.e. in regime III.

If the condition $c>0$ is satisfied, the fixed points of the minimal model (i.e. the zeros of $R_{\mathrm{nf}}$ ) reproduce the three dynamical regimes observed for the full model (see Fig. 4(a)). Regime I (non-active) is bounded from above by a line $a=0$ (for $b<0$ ) of TC bifurcations and by a parabolic line $a=-b^{2} / 4 c$ (for $b<0$ ) of SN bifurcations. Regime II (active) is observed for any $a>0$, i.e. above the TC line which exists for any value of $b$. Regime III (bi-stable) is bounded by the TC bifurcation line from above and by the SN bifurcation line from below (i.e. it exists provided $a<0$ and $b>2 \sqrt{-c a}$ ). All the three regimes meet at the co-dimension two SNT crossing bifurcation point located at $a=b=0$.

In fact, the minimal model with $c>0$ exhibits all three regimes on the plane $(\alpha, \beta)$ corresponding to the full model. In order to prove this, it is necessary to show that the domain in the $(\alpha, \beta)$ parameter space where $c>0$ (with $c$ given by Eq. (23)) covers a finite neighbourhood of the SNT crossing bifurcation point where the three regimes meet. In Appendix Appendix B, we prove this for c-time dynamics. A rigorous proof for d-time dynamics is more challenging but numerical analyses suggest that this also holds for d-time dynamics on random regular graphs with any $k$.

The minimal model leads to several interesting conclusions. First, it shows that the co-dimension of the SNT crossing bifurcation is 2. Second, it demonstrates that considering synergistic effects associated with up to 3 neighbours is sufficient to observe three regimes for synergistic invasions on random regular graphs with any degree $k$. This follows from the fact that the coefficients $a, b$ and $c$ depend only on the transmission rates $\Lambda_{1}, \Lambda_{2}$ and $\Lambda_{3}$. Synergistic effects associated with more than 3 neighbours may play a role on the details of invasions but do not affect their qualitative behaviour. 

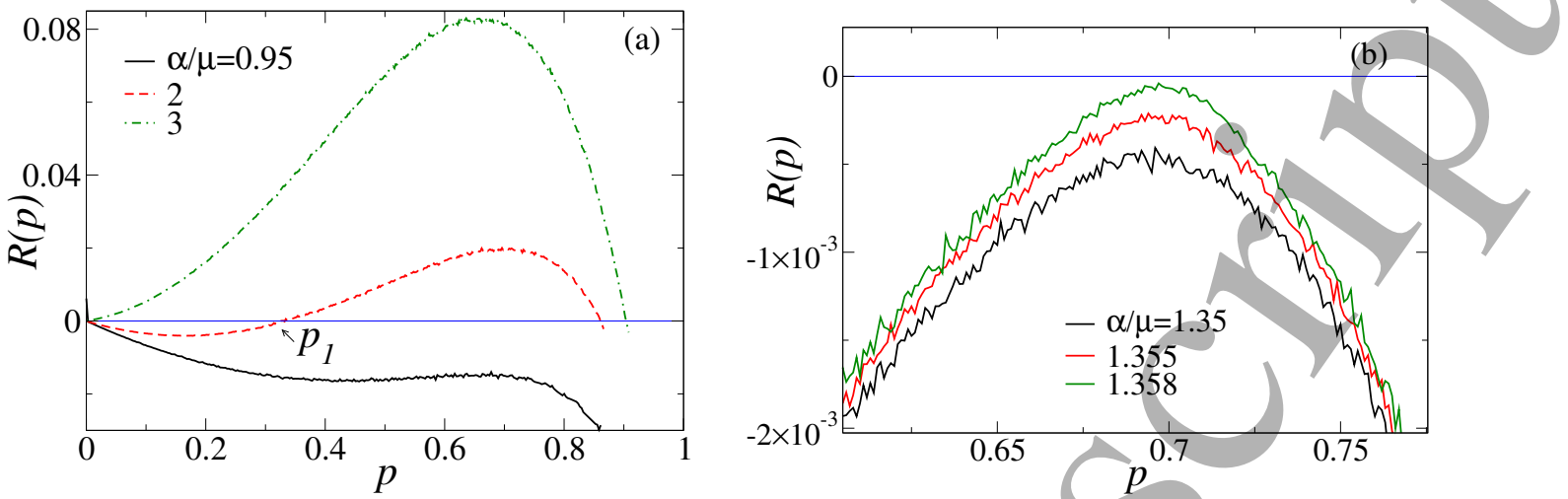

Figure 5. The dependence of the rate function, $R(p)$, calculated numerically on concentration of infected nodes, $p$, for d-time SIS process exhibiting S-synergy in transmission on random 10-regular graphs $\left(N=10^{5}\right)$ with $\mu=0.1, \beta=-0.4$ and different values of $\tilde{\alpha}=\alpha / \mu$ as indicated in the legends. The panel (a) shows $R(p)$ for non-active (solid line, $p(0)=1$ ), bi-stable (dashed, $p(0)=0.307$ ) and active (dotdashed, $p(0)=10^{-3}$ ) regimes. The panel (b) displays $R(p)$, for values of $\tilde{\alpha}$ near the SN bifurcation $(p(0)=1$ for all the curves).

\section{Numerical simulations}

The above analysis performed within the single-site mean-field approximation is inherently inexact and thus needs to be validated by exact numerical simulations. For bifurcation analysis, it appears convenient to calculate numerically the rate function $R(p)$ entering Eq. (2) and investigate the behaviour of its roots with variation of parameters of the model. The advantage in analysing numerically the rate function rather than just calculating the time series for concentration of infected nodes is the following. It gives unambiguous criteria for location of (i) the SN bifurcations, i.e. discontinuous transitions in concentration of infected nodes, and (ii) unstable equilibrium points (cf. $\{9,36,47]$ ). The rate function can be used for location of the TC bifurcations as well although the procedure for finding critical points for continuous transitions is well established [16, 18].

The shape of the rate function calculated numerically follows that predicted by single-site mean-field analysis (cf. the curves shown in Fig. 1(c) and Fig. 5(a) shown for SIS processes exhibiting S-synergy). As follows from Fig. 5(a), the roots of the rate function (and thus the concentration of infected nodes in stable and unstable equilibrium states) can be identified. This is particularly important for the roots corresponding to unstable equilibria (see the middle root at $p_{1} \simeq 0.33$ for the dashed curve in Fig. 5(a)). The range of $p$ for which the rate function in bi-stable regime is available numerically significantly depends on initial conditions, i.e. on $p(0)$. If $p(0) \simeq p_{1}$, then the SIS process can either go to extinction or invasion and $R(p)$ becomes available for practically the whole range of concentration of infected nodes. In particular, the dashed curve shown in Fig. 5(a) is calculated for $p(0)=0.307$ averaged over $10^{4}$ network configurations resulting in $p_{1} \simeq 0.339 \pm 0.007$. 
The location of the SN bifurcation can be estimated by finding the values of the rate function at the maximum for different values of $\tilde{\alpha}$ and extrapolating them to zero. An example of evolution of $R(p)$ near the SN bifurcation is shown in Fig. 5(b) leading to an estimate for $\tilde{\alpha}_{\mathrm{SN}} \simeq 1.3595 \pm 0.0005$.

Overall, we found that numerical analysis supports qualitatively all the main findings within a simple single-site mean-field approximation. As expected, the quantitative agreement between $\mathrm{MC}$ and mean-field data are not perfect. Indeed, comparing Figs. 1(c) and Fig. 5(a), we can see that the evolution of the shape of the rate function with $\tilde{\alpha}$ is similar for both mean-field and MC data but the values of $R(p)$ for the same $\tilde{\alpha}$ are quite different in both panels (cf. e.g. solid lines for $\tilde{\alpha}=0.95$ ). Consequently, the open symbols representing MC data in Figs 1(a) and 3(a) deviate from the continuous bifurcation lines obtained analytically. The disagreement between $\mathrm{MC}$ and mean-field data are sufficiently significant in the location of SN bifurcations for very negative values of $\beta$ in the case of S-synergy (cf. location of squares for MC data and dashed lines for mean-field in Fig. 1(a) and, especially, in Fig. 2(a) for 3-regular graph).

This is not surprising because the single-site mean-field approximation completely ignores the dynamical correlations which can be quite important for synergistic SIS processes. However, the main qualitative features such as existence of three different regimes are well reproduced by single-site mean-field analysis.

Several approaches have been developed for dealing with dynamical correlations in synergy-free spreading processes $[5,10,11,17,20,31,34]$. The synergy effects bring new features in the dynamics which do not permit to use straightforwardly the results obtained for synergy-free cases. A possible way forward could consist in using a two-site approximation for $P\left(C_{n}\right)$ in Eq. (4) which we hope to address in future analysis.

\section{Conclusions}

To conclude, we presented the single-site mean-field analysis for synergistic SIS processes spreading on random k-regular graphs. The synergy effects investigated account for possible non-linear cooperative effects in transmission of infection between nodes in a network. In particular, two cases were investigated in which the individual rate of transmission from an infected node to a susceptible one depends on number of neighbours of susceptible node being either in susceptible (S-synergy) or infected (Isynergy) states. The synergistic transmission is parameterised by introducing two parameters, the inherent transmission rate (i.e. the rate in the synergy-free limit) and the strength of synergy, in such a way that the synergistic transmission rates are continuous functions of both of these parameters and also of discrete number of infected neighbours affecting the transmission. The latter property distinguishes the synergy model from the popular threshold models. Moreover, the continuity of the synergistic rates in all variables makes possible to analyse phase diagrams in two-parameter space and reveal quite a rich picture even in the simplest topological case of k-regular graphs. 


\section{Bifurcations in synergistic epidemics}

In particular, the phase diagrams for synergistic SIS process on k-regular graphs (with $k>2$ ) exhibit three regions found both numerically and analytically: non-active, active and bi-stable. These regions are separated by lines of transcritical and saddle-node bifurcations which cross (interact) at the saddle-node-transcritical bifurcation. The latter bifurcation point, to our knowledge, has not been observed within the threshold models.

Also, we developed a numerical procedure based on analysis of the rate function for detection of the saddle-node bifurcations, transcritical bifurcations at the boundary between stable and active regimes and unstable equilibria in bi-stable regime. In these cases, the standard analysis of the time-series for concentration of infected nodes is not very helpful. In contrast, analysis of the rate of change in concentration of infected nodes appears to be sufficiently revealing.

The synergy model is general and can be applied to various spreading processes (e.g. SI, SIS, SIR, contact, catalytic reaction-diffusion and others) on networks of different topology. Our preliminary analysis of synergistic processes on other networks (Erdös-Rényi, binary and scale-free) shows even more complex bifurcation diagrams with possibility of appearance of several SNT, SN, TC and cusp bifurcations. Currently, the synergy effects are analysed only for transmission rates but they can be straightforwardly incorporated for recovery rates as well. Analytically, the main challenge remains in accurate description of the dynamical correlations for synergistic processes, which we hope to address in the future.

\section{Acknowledgments}

Acknowledgements FJPR acknowledges financial support from the Carnegie Trust.

\section{Appendix A. Stability of fixed points}

In this section, we study the stability of fixed points of the solutions of the proposed models in the single-site mean-field approximation which obey Eq. (2) with the rate function $R(p)$ given by Eq. (7). Stability of fixed points can be qualitatively understood from the graphical representation of $R(p)$ but here we present a more rigorous analysis based on the Lyapunov function method $[1,4]$.

Before dealing with the synergistic epidemic model, consider a generic timeinvariant dynamical system described by a variable $x \in \mathbb{R}$ which obeys d-time dynamics given by the difference equation,

$$
x(t+\delta t)=x(t)+f(x(t)) \delta t .
$$

Assume that the real-valued function $f(x)$ satisfies

$$
f(x)= \begin{cases}>0 & \text { for } x \in\left(a, x^{*}\right) \\ =0 & \text { for } x=x^{*} \\ <0 & \text { for } x \in\left(x^{*}, b\right)\end{cases}
$$




\section{Bifurcations in synergistic epidemics}

in some interval $(a, b)$ of $x$, where $a$ and $b$ are real parameters, $a<b$.

Theorem Appendix A.1 The point $x=x^{*}$ is an asymptotically stable fixed point in the interval $(a, b)$.

Proof. The function

$$
V(x)=-\int_{x^{*}}^{x} f(y) d y
$$

is positive definite for any value of $x \in(a, b)$ except at $x=x^{*}$ where it is $V\left(x^{*}\right)=0$.

In addition, the variation of $V(x)$ with time along any trajectory $x(t)$ of the system,

$$
\frac{\delta V}{\delta t}=\frac{V(x(t+\delta t))-V(x(t))}{\delta t}=-\frac{1}{\delta t} \int_{x(t)}^{x(t)+f \delta(t)} f(y) d y
$$

is negative everywhere in $(a, b)$ except at $x=x^{*}$.

Indeed, for $x \in\left(x^{*}, b\right), f(x)<0$ and one obtains

$$
\delta V=-\int_{x(t)}^{x(t)+|f(x(t))| \delta t}|f(y)| d y<0
$$

Similarly, for $x \in\left(a, x^{*}\right), f(x)>0$ and one obtains

$$
\delta V=-\int_{x(t)-|f(x(t))| \delta t}^{x(t)}|f(y)| d y<0 .
$$

This proves that $V(x)$ is a Lyapunov function and $x=x^{*}$ is an asymptotically stable fixed point in the interval $(a, b)$.

Corollary Appendix A.1.1 In the c-time limit (i.e. for infinitesimal $\delta t \rightarrow d t$ ), the system (A.1) has a fixed point at $x=x^{*}$ which is asymptotically stable in $(a, b)$.

Proof. In the c-time limit, the difference equation (A.1) reduces to $d x / d t=f(x)$ and the variation of $V(x)$ with respect to time along a trajectory $x(t)$ of the system is

$$
\frac{d V}{d t}=-(f(x(t)))^{2}<0
$$

for any $x \in(a, b)-\left\{x^{*}\right\}$. Therefore, $V(x)$ is a Lyapunov function and this proves the corollary.

We now use these results to analyse the stability of fixed points for the concentration of infected nodes, $p(t)$, given by Eq. (2) with $R(q)$ given by Eq. (7). The results apply in general to both $\mathrm{S}$ - and I-synergy models with both d- and c-time dynamics.

Corollary Appendix A.1.2 (Stability in regime I) Consider regime $I$ which is characterised by a single fixed point at $p=p_{0}=0$. This fixed point is globally asymptotically stable in the feasible interval of $p \in(0,1]$.

Proof. Since $p_{0}=0$ is the only fixed point and the infection rate satisfies $R(0)=0$ and $R(1)<0$, it is clear that $R(p)<0$ for any $p \in(0,1]$. For c-time dynamics, this system is equivalent to the system given by Eq. (A.1) with $x \in\left(x^{*}, b\right)$. Accordingly, $p_{0}=0$ is globally asymptotically stable in $(0,1]$ by Theorem Appendix A.1. Similarly, stability in the d-time case follows from corollary Appendix A.1.1. 


\section{Bifurcations in synergistic epidemics}

Corollary Appendix A.1.3 (Stability in regime II) In regime II, there are the non-active $\left(p=p_{1}>0\right)$ and active $\left(p=p_{0}=0\right)$ fixed points. The active fixed point, $p_{1}>0$, is globally asymptotically stable in the feasible interval $(0,1]$ and the non-active fixed point $p_{0}=0$ is unstable.

Proof. Let us first consider the fixed point for the active regime. The conditions $R(0)=0$ and $R(1)<0$ imply that $R(p)>0$ for $p \in\left(0, p_{1}\right)$ and $R(p)<0$ for $p \in\left(p_{1}, 1\right]$. Therefore, the problem reduces to that of the system A.1 with $a=0, x^{*}=p_{1}$ and $b=1$. By Theorem Appendix A.1 and Corollary Appendix A.1.1 it is clear that the fixed point at $p=p_{1}$ is globally asymptotically stable in the feasible interval $(0,1]$ for both $\mathrm{d}$ - and c-time dynamics. The fixed point at $p_{0}=0$ corresponding to the non-active regime is therefore unstable.

Corollary Appendix A.1.4 (Stability in regime III) In regime III, there are three fixed points: the non-active fixed point at $p=p_{0}=0$, the active fixed point at $p=p_{2}>0$ and a fixed point with intermediate $p=p_{1} \in\left(p_{0}, p_{2}\right)$. Stability of these points is as follows for both $d$ - and c-time dynamics:

(i) The fixed point at $p=p_{0}=0$ is locally asymptotically stable in the interval $\left(0, p_{1}\right)$.

(ii) The fixed point at $p=p_{1}$ is unstable.

(iii) The fixed point at $p=p_{2}$ is locally asymptotically stable in the interval $\left(p_{1}, 1\right]$.

Proof. Local stability of $p=p_{0}=0$ and $p=p_{2}$ follows from Theorem Appendix A.1 and Corollary Appendix A.1.1 using the conditions $R(0)=0$ and $R(1)<0$ which imply the following behaviour for $R(p)$ :

$$
R(p)=\left\{\begin{array}{cc}
<0 & \text { for } p \in\left(0, p_{1}\right) \\
>0 & \text { for } p \in\left(p_{1}, p_{2}\right) \\
<0 & \text { for } p \in\left(p_{2}, 1\right] .
\end{array}\right.
$$

Since the basins of attraction of the fixed points $p=p_{0}=0$ and $p=p_{2}$ cover the whole feasible interval $(0,1]$ except for the point $p=p_{1}$, we conclude that the fixed point at $p=p_{1}$ is unstable.

\section{Appendix B. Validity of the minimal model}

In this appendix, we show for c-time that the condition $c>0$ (see Eq. (23)) defines a region in the $(\alpha, \beta)$ parameter space which contains the SNT crossing bifurcation for both S- and I-synergy if $k>2$.

The transmission rate for c-time dynamics reduces to $\Lambda_{n}=n \lambda_{n}$ and this allows the condition $c>0$ to be expressed as follows:

$$
(k-2) \lambda_{3}-2(k-1) \lambda_{2}+k \lambda_{1}<0 .
$$




\section{Bifurcations in synergistic epidemics}

This condition is satisfied for any value of $\alpha$ and $\beta \in B$, where

$$
B= \begin{cases}\left(-\ln \left(\frac{\mathrm{k}}{\mathrm{k}-2}\right), 0\right) & \text { for S-synergy } \\ \left(0, \ln \left(\frac{\mathrm{k}}{\mathrm{k}-2}\right)\right) & \text { for I-synergy }\end{cases}
$$

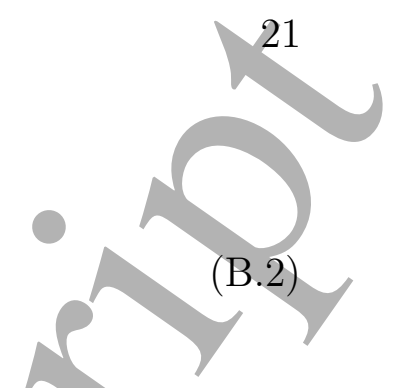

From Eqs. (12) and (16) it is clear that the SNT crossing bifurcation point belongs to $B$ for both S- and I-synergy.

\section{References}

[1] Arnol'd, V.I.: Ordinary Differential Equations. Springer-Verlag Berlin Heidelberg, Heidelberg (1992)

[2] Barrat, A., Barthélemy, M., Vespignani, A.: Dynamical Processes on Complex Networks. Cambridge University Press, Cambridge (2008)

[3] Baxter, G.J., Dorogovtsev, S.N., Goltsev, A.V., Mendes, J.F.F.: Bootstrap percolation on complex networks. Phys. Rev. E 82, 011103 (2010)

[4] Bof, N., Carli, R., Schenato, L.: Lyapunov Theory for Diserete Time Systems. Tech. rep., Universita degli studi di Padova (2018). URL http://arxiv.org/abs/1809.05289

[5] Boguñá, M., Castellano, C., Pastor-Satorras, R.: Nature of the Epidemic Threshold for the Susceptible-Infected-Susceptible Dynamics in Networks. Phys. Rev. Lett. 111, 068701 (2013)

[6] Böttcher, L., Lukovic, M., Nagler, J., Havlin, S., Herrmann, H.J.: Bootstrap percolation on complex networks. Sci. Rep. 7, 41729 (2017)

[7] Böttcher, M., Nagler, S., Herrmann, H.J.: Critical Behaviors in Contagion Dynamics. Phys. Rev. Lett. 118, 088301 (2017)

[8] Bollobás, B.: Random Graphs. Cambridge University Press, Cambridge (2001)

[9] Chae, H., Yook, S.H., Kim, Y.: Discontinuous phase transition in a core contact process on complex networks. New J. of Physics 17, 023039 (2015)

[10] Cai, C.-R., Wu, Z.-X., Guan, J.-Y.: Effective degree Markov-chain approach for discrete-time epidemic processes on uncorrelated networks. Phys. Rev. E 90, 052803 (2014)

[11] Cai, C.-R., Wu, Z.-X., Chen, M. Z. Q., Holme, P., Guan, J.-Y.: Solving the Dynamic Correlation Problem of the Susceptible-Infected-Susceptible Model on Networks. Phys. Rev. Lett. 116, 258301 (2016)

[12] Centola, D.: The Spread of Behavior in an Online Social Network Experiment. Science 329, 1194 (2010)

[13] Dodds, P.S., Watts, D.J.: Universal behavior in a generalized model of contagion. Phys. Rev. Lett. 92, 218701 (2004)

[14] Dorogovtsev, S. N., Goltsev, A. V.: Critical phenomena in complex networks. Rev. Mod. Phys. 80, 1275-1335 (2008).

[15] van den Driessche, P., Watmough, J.: Reproduction numbers and sub-threshold endemic equilibria for compartmental models of disease transmission. Math. Biosci. 180(1-2), 29-48 (2002)

[16] Ferreira, S.C. Castellano, C., Pastor-Satorras, R.: Epidemic thresholds of the susceptible-infectedsusceptible model on networks: A comparison of numerical and theoretical results. Phys. Rev. E 86, 041125 (2012)

[17] Ferreira, R.S., Ferreira, S.C.: Critical behavior of the contact process on small-world networks. Eur. Phys. J. B 86, 462 (2013)

[18] Ferreira, S.C., Ferreira, R.S., Castellano, C., Pastor-Satorras, R.: Quasistationary simulations of the contact process on quenched networks. Phys. Rev. E 84, 066102 (2011)

[19] Gleeson, J.P., Melnik, S., Ward, J. A., Porter, M. A., Mucha, P. J.: Accuracy of Mean-Field Theory for Dynamics on Real-World Networks, Phys. Rev. E 85, 026106 (2012). 


\section{Bifurcations in synergistic epidemics}

[20] Gleeson, J.P.: Binary-state dynamics on complex networks: Pair approximation and beyond. Phys. Rev. X 3, 021004 (2013)

[21] Gomez-Gardenes, J., Lotero, L., Taraskin, S., Perez-Reche, F.: Explosive Contagion in/Networks. Sci. Rep. 6, 19767 (2015)

[22] Gross, T., D'Lima, C.J.D., Blasius, B.: Epidemic dynamics on an adaptive network. Phys. Rev. Lett. 96, 208701 (2006)

[23] Gubbins, S., Gilligan, C.A., Kleczkowski, A.: Population dynamics of plant-parasite interactions: Thresholds for invasion. Theor. Popul. Biol. 57, 2000 (2000)

[24] Guo, D., Trajanovski, S., van de Bovenkamp, R., Wang, H., Van Mieghem, P.: Epidemic threshold and topological structure of susceptible-infectious-susceptible epidemics in adaptive networks. Phys. Rev. E 88, 042802 (2013)

[25] Hethcote, H.W.: The Mathematics of Infectious Diseases. SIAM Rev. 42(4), 599 (2000)

[26] Juul, J.S., Porter, M.A.: Synergistic effects in threshold models on networks. Chaos 28, 013115 (2018)

[27] Liu, D.-J., Guo, X., Evans, J.W.: Quadratic Contact Process: Phase Separation with InterfaceOrientation-Dependent Equistability Phys. Rev. Lett. 98, 050601 (2007)

[28] Liu, Q.H., Wang, W., Tang, M., Zhou, T., Lai, Y.C.: Explosive spréading on complex networks: The role of synergy. Phys. Rev. E 95, 042320 (2017)

[29] Liu, W.m., Hethcote, H.W., Levin, S.A.: Dynamical behavior of epidemiological models with nonlinear incidence rates. J. Math. Biol. 25(4), 359-380 (1987)

[30] Ludlam, J.J, Gibson, G. J., Otten, W., Gilligan, C.A.: Applications of percolation theory to fungal spread with synergy. J. R. Soc. Interf. 9, 949 (2011).

[31] Luo, X.F., Zhang, X.G., Sun, G.Q., Jin, Z.: Epidemical dynamics of SIS pair ap- proximation models on regular and radnom networks. Physica A 410, 144 (2014)

[32] Marceau, V., Noël, P.A., Hébert-Dufresne, L., Allard, A., Dubé, L.J.: Adaptive networks: Coevolution of disease and topology. Phys. Rev. E 82, 036116 (2010)

[33] Majdandzic, A., Podobnik, B., Buldyrev, S. V., Kenett, D. Y., Havlin, S., Stanley, E. H.: Spontaneous recovery in dynamical networks. Nature Phys. 10, 34 (2014)

[34] Mata, S.A., Ferreira, R.S., Ferreira, S.C.: Heterogeneous pair-approximation for the contact process on complex networks. New J. Phys. 16, 053006 (2014)

[35] Min, B., Miguel, M.S.: Competing contagion processes: Complex contagion triggered by simple contagion. Sci. Rep. 8,10422 (2018)

[36] de Oliveira, M.M., da Luz, M.G.E., Fiore, C.E.: Generic finite size scaling for discontinuous nonequilibrium phase transitions into absorbing states. Phys. Rev. E 92, 062126 (2015)

[37] Pastor-Satorras, R., Castellano, C., Van Mieghem, P., Vespignani, A.: Epidemic processes in complex networks. Rev. Mod. Phys. 87, 925 (2015)

[38] Pérez-Reche, F.J., Ludlam, J.J., Taraskin, S.N., Gilligan, C.A.: Synergy in spreading processes: From exploitative to explorative foraging strategies. Phys. Rev. Lett. 106, 218701 (2011)

[39] Pianegonda, S., Fiore, C.E.: Effect of diffusion in simple discontinuous absorbing transition models. J. Stat. Mech. P08018 (2015)

[40] Porter, M. A., Gleeson, J. P.: Dynamical Systems on Networks. Springer International Publishing, Cham (2016)

[41] Schlögl, F.: Chemical reaction models for non-equilibrium phase transitions. Z. Phys. 253, 147 (1972)

[42] Saputra, K., Veen, L., Quispel, G.: The saddle-node-transcritical bifurcation in a population model with constant rate harvesting. Discret. Contin. Dyn. Syst. - Ser. B 14, 233 (2010)

[43] da Silva, E. F., de Oliveira, M.M.: Critical discontinuous phase transition in the threshold contact process. J. Phys. A: Math. Theor. 44, 135002 (2011)

[44] Strogatz, S.: Nonlinear Dynamics and Chaos. Addison-Wesley, Reading, Massachusetts (1994)

[45] Taraskin, S.N., Pérez-Reche, F.J.: Effects of variable-state neighborhoods for spreading synergystic processes on lattices. Phys. Rev. E 88, 062815 (2013) 
1

2

3

4

5

6

7

8

9

10

11

12

13

14

15

16

17

18

19

20

21

22

23

24

25

26

27

28

29

30

31

32

33

34

35

36

37

38

39

40

41

42

43

44

45

46

47

48

49

50

51

52

53

54

55

56

57

58

59

60

Bifurcations in synergistic epidemics

[46] Watts, D. J.: A simple model of global cascades on random networks. Proc. Natl Acad. Sci. USA 99, 5766-5771 (2002)

[47] Varghese, C., Durrett, R.: Phase transitions in the quadratic contact process on complex networks. Phys. Rev. E 87, 062819 (2013)

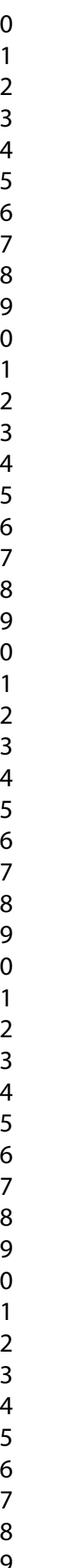

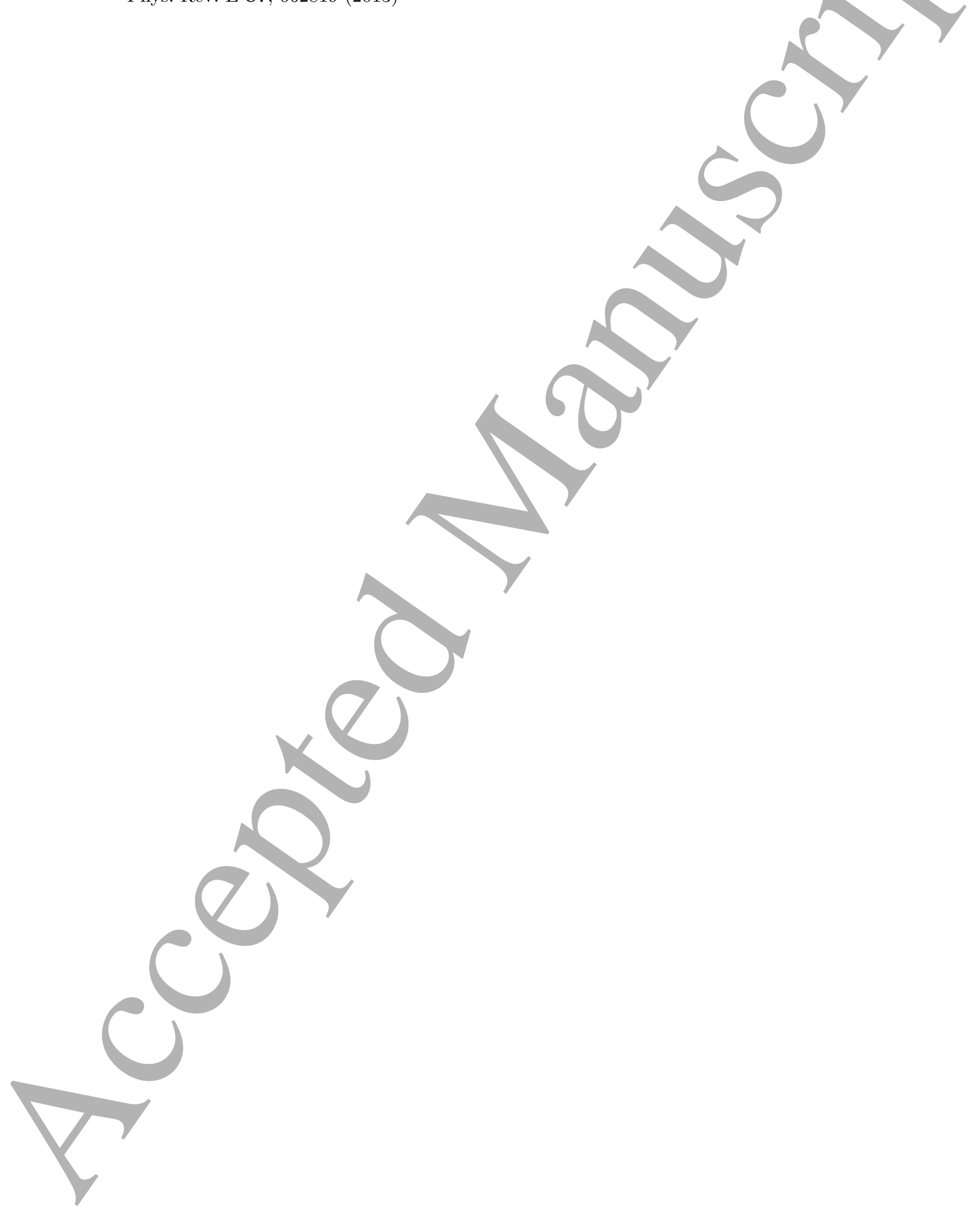

